\title{
Research Article \\ PP- and Split PS-Wave AVA Responses of Fractured Shale
}

\author{
Shuai Yang $(\mathbb{B}$ and Jun Lu $(1)$ \\ Key Laboratory of Marine Reservoir Evolution and Hydrocarbon Accumulation Mechanism, Ministry of Education, \\ China University of Geosciences, Beijing 100083, China \\ Correspondence should be addressed to Jun Lu; lujun615@163.com
}

Received 18 December 2017; Revised 24 March 2018; Accepted 19 April 2018; Published 17 May 2018

Academic Editor: Xiao-Qiao He

Copyright ( $) 2018$ Shuai Yang and Jun Lu. This is an open access article distributed under the Creative Commons Attribution License, which permits unrestricted use, distribution, and reproduction in any medium, provided the original work is properly cited.

With seismic exploration advancing to the deep Earth, the seismic response of fractured strata has currently become a hot research topic. However, characteristics of the amplitude variation with angle (AVA) of saturated fractured shale still remain unclear. Furthermore, the direct relationships between the AVA response and fracture system parameters have not received much attention. This study is aimed at analyzing the effect of fracture density on AVA responses of fractured shale. For this purpose, we propose a method for modeling saturated fractured shale and analyzing AVA responses of PP- and split PS-waves. First, we introduce Gurevich's fluid theory into the fractured-shale modeling and establish the relationship between Thomsen's weak anisotropy parameters and fracture density and fluid properties. Second, we perform forward simulation considering an isotropic overburden and a fractured stratum. The results show that differences in AVA responses of the fractured-shale model and the isotropic model increase with increasing fracture densities. At small to intermediate incidence angles, the reflection coefficients of split PS-waves increase, whereas those of PP-wave decrease. The reflection coefficients of the two models differ dramatically at incidence angles larger than $55^{\circ}$. Furthermore, when the fracture density is large, polarity reversal occurs only in PS2-wave AVA gathers.

\section{Introduction}

With the recent intensification of exploration and development of unconventional oil and gas reservoirs, geophysical prospecting methods of shale reservoirs have been receiving much attention worldwide. Among such methods, the AVO (amplitude variation with offset) or AVA (amplitude variation with angle) technique is considered as one of the most promising techniques as it has played an important role in conventional oil and gas exploration. Castagna et al. [1] studied AVO intercepts and gradients for pairs of shale/gas sand. Avseth et al. [2] derived the shale trend for a gas-and-oil field of North Sea through rock physics modeling and AVO analysis. However, thus far AVO studies of shales have only considered isotropic media and ignored seismic anisotropy, leading to deviations in shale reservoir characterization. In fact, due to the compaction of the overburden, high-angle fractures are widely developed in shale reservoirs, which serve as important reservoir spaces and seepage channels for shale gas. Because of tectonic stress, high-angle fractures tend to be aligned along the same direction. Such reservoir models can be described as a kind of anisotropic media named HTI media (transversely isotropic media with a horizontal axis of symmetry) [3]. There are three modes of body wave propagation in this media: a P-wave, two shear-waves with different velocities, and mutually orthogonal polarization directions $[4,5]$. These two shear-waves are split from the same shear wave when its polarization direction is oblique to the fracture plane.

Some scholars have studied the AVO responses of HTI media. However, these studies mainly focused on the application of $\mathrm{P}$-wave AVO responses to the prediction of fractured reservoirs. Rüger and Tsvankin [6,7] used the P-wave azimuthal AVO method to detect fractures. Rüger $[8,9]$ further extended the P-wave reflection coefficient to HTI media. Pérez et al. [10] studied the practical application of Pwave AVA to the detection of fracture orientation. Gray and Head [11] studied the properties of anisotropic media based on the P-wave azimuthal anisotropy and predicted fractures in an actual oilfield. Hall and Kendall [12] applied the Pwave AVA of a submarine three-component seismic dataset to analyze natural fracture characteristics. Rüger $[13,14]$ 


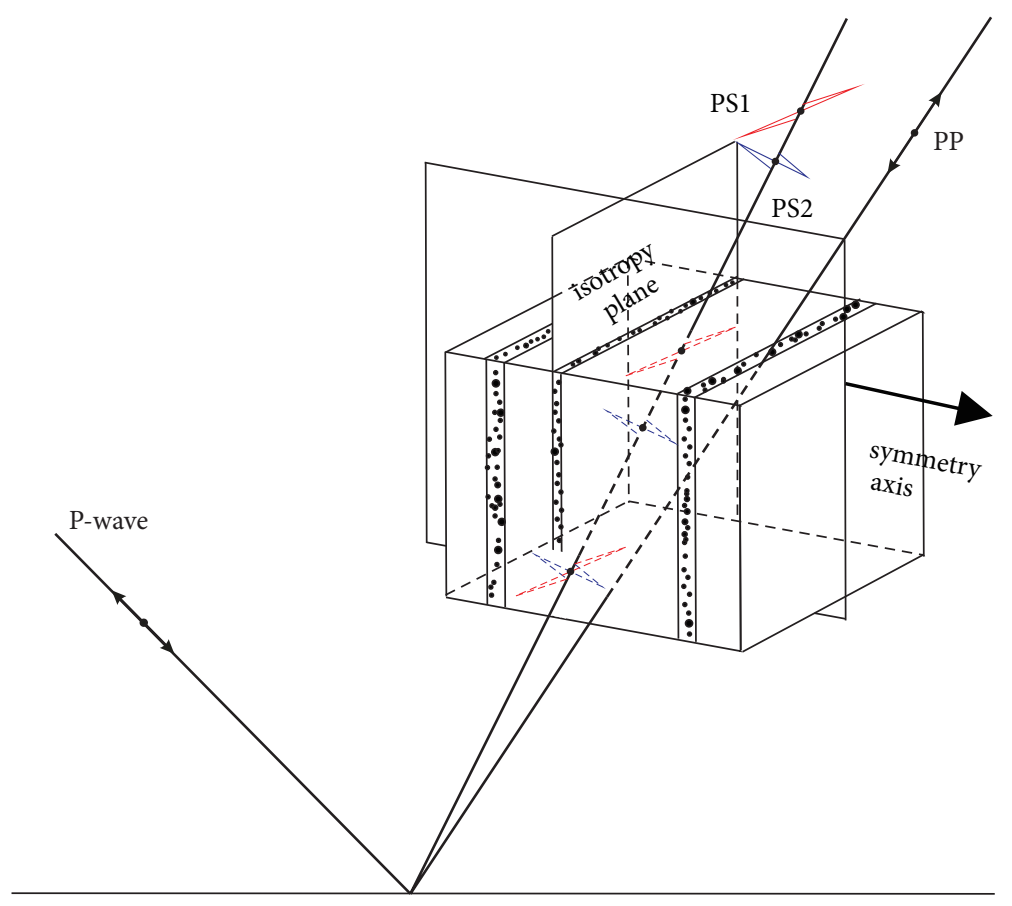

FIGURE 1: Schematic of the HTI model.

proposed the approximate reflection coefficient equations for HTI media using Thomsen's anisotropy parameters.

Previous AVO researches on fractured reservoirs are mainly based on commonly used fracture anisotropy models: Hudson model [15] and linear slip model [16]. However, both models assume that the background medium embedded in the fracture is a single elastic solid medium without considering pores and fluids. In reality, the presence of pore fluids and wave-induced fluid flow between pores and fractures strongly influence the propagation of elastic waves. Recent studies have proposed an HTI model that considers the properties of both fluid flow and seismic anisotropy. Thomsen $[17,18]$ proposed the theories of weak seismic anisotropy and used five parameters to characterize reservoir anisotropy. Thomsen considered fractured rocks as an isotropic model containing a series of fractures connected with the same pore diameter, and fluids flow between the fractures and pores. This theory is more applicable for computing the seismicwave characteristics of the fluid-filled fracture model in the seismic scale. Chapman [19] proposed the fracture models with both pore and fracture dimensions. Cardona [20] used the anisotropic Gassmann equations to achieve a fluid independent replacement of the geometrical shape of fractures. Gurevich [21] further developed the formula of the fluid substitution interpretation for the vertical fractured media with the fluids in pores. Gurevich's formula can establish the exact analytical relationships between the effective stiffness matrix of a rock and the elastic properties of the dry background, porosity, fracture compliances, and the saturating fluid. However, studies on the direct relationship between seismic-wave responses and fracture system parameters, such as fracture density and fillings, are lacking.
In this study, based on Gurevich's theory of saturating fluid, we established the relationship between Thomsen's anisotropy and elastic parameters and fracture system parameters. Both fluidity and the anisotropy were considered in the proposed HTI model. Then, we built a theoretical fracturedshale model and applied the reflection coefficient equations derived by Rüger to analyze PP- and split PS-wave AVA responses.

\section{Methodology}

2.1. Mathematical Model. There are four common anisotropic media in the Earth [22]. Triclinic medium has no symmetry planes or an axis of cylindrical symmetry; monoclinic medium has only a symmetry plane; orthorhombic medium has three mutually orthogonal planes of symmetry; transversely isotropic medium has an axis of cylindrical symmetry. Transversely isotropic medium is the simplest anisotropic medium, which is referred to as the HTI medium when the symmetry axis is horizontal. The anisotropy of an HTI medium in the oil and gas exploration is always induced by a single fracture set embedded in an isotropic matrix [14]. Therefore, we used the HTI model to establish single-layer shale with aligned vertical fractures as the model illustrated in Figure 1. The fine layering model with vertical fractures was not considered, because it involves a more complex anisotropic model with multigroup and multiscale fractures.

In a multicomponent seismic survey, $\mathrm{P}$-wave sources are used to initiate seismic waves. As shown in Figure 1, the reflected $\mathrm{P}$-wave ( $\mathrm{PP}$-wave) propagates through the fracture zone without changing the original polarization direction. When the original polarization direction is oblique to the 
fracture (isotropy) plane, the reflected S-wave (PS-wave) splits into a fast mode (PS1-wave) and a slow mode (PS2wave). The polarization directions of the split PS1- and PS2-waves are parallel and vertical to the fracture plane, respectively. In the HTI model, the elastic properties of the fractured shale can be described using the following stiffness coefficient matrix [14]:

$$
\mathbf{C}=\left[\begin{array}{cccccc}
c_{11} & c_{13} & c_{13} & 0 & 0 & 0 \\
c_{13} & c_{33} & \left(c_{33}-2 c_{44}\right) & 0 & 0 & 0 \\
c_{13} & \left(c_{33}-2 c_{44}\right) & c_{33} & 0 & 0 & 0 \\
0 & 0 & 0 & c_{44} & 0 & 0 \\
0 & 0 & 0 & 0 & c_{55} & 0 \\
0 & 0 & 0 & 0 & 0 & c_{55}
\end{array}\right] .
$$

Elastic and anisotropy parameters related to AVA responses can be converted by the stiffness coefficients:

$$
\begin{aligned}
\alpha & =\sqrt{\frac{c_{33}}{\rho}}, \\
\beta & =\sqrt{\frac{c_{44}}{\rho}}, \\
\varepsilon^{(V)} & =\frac{c_{11}-c_{33}}{2 c_{33}}, \\
\delta^{(V)} & =\frac{\left(c_{13}+c_{55}\right)^{2}-\left(c_{33}-c_{55}\right)^{2}}{2 c_{33}\left(c_{33}-c_{55}\right)}, \\
\gamma^{(V)} & =\frac{c_{55}-c_{44}}{2 c_{44}}, \\
\gamma & =-\frac{2 \gamma^{(V)}+1}{\gamma^{(V)}},
\end{aligned}
$$

where the elastic parameters $\alpha, \beta$, and $\rho$ denote the isotropyplane velocities of the vertical P- and S1-waves and density, respectively; $\varepsilon^{(V)}$ and $\delta^{(V)}$ are the anisotropy parameters characterizing the P-wave velocity anisotropy; $\gamma^{(V)}$ is the anisotropy parameter indicating the fractional difference between the velocities of S2- and S1-waves. The superscript " $V$ " denotes vertical direction along the fracture plane.

Regarding saturated fractured shale, individual fluid substitution expressions for each stiffness coefficient of the HTI model are as follows $[21,23]$ :

$$
\begin{aligned}
& c_{11}^{\text {sat }}=\frac{L}{D}\left[d_{1} \eta+\frac{K_{f}}{\varphi K_{m} L}\left(L_{1} b^{\prime}-\frac{16 \mu^{2} b_{0} \Delta_{N}}{9 L}\right)\right], \\
& c_{33}^{\text {sat }}=\frac{L}{D}\left[d_{2} \eta+\frac{K_{f}}{\varphi K_{m} L}\left(L_{1} b^{\prime}-\frac{4 \mu^{2} b_{0} \Delta_{N}}{9 L}\right)\right], \\
& c_{13}^{\text {sat }}=\frac{\lambda}{D}\left[d_{1} \eta+\frac{K_{f}}{\varphi K_{m} \lambda}\left(\lambda_{1} b^{\prime}-\frac{8 \mu^{2} b_{0} \Delta_{N}}{9 L}\right)\right], \\
& c_{44}^{\text {sat }}=\mu, \\
& c_{55}^{\text {sat }}=\mu\left(1-\Delta_{T}\right),
\end{aligned}
$$

where

$$
\begin{aligned}
& L=\lambda+2 \mu, \\
& D=1+\frac{K_{f}}{K_{m} \varphi}\left(b_{0}-\varphi+\frac{K^{2} \Delta_{N}}{K_{m} L}\right) \text {, } \\
& \eta=1-\frac{K_{f}}{K_{m}} \\
& \varphi=\varphi_{m}\left(1-\varphi_{c}\right)+\varphi_{c}, \\
& \varphi_{c}=\frac{4 \pi a e}{3}, \\
& b_{0}=1-\frac{K}{K_{m}}, \\
& b^{\prime}=b_{0}+\frac{K^{2} \Delta_{N}}{K_{m} L}, \\
& L_{1}=K_{m}+\frac{4}{3} \mu \text {, } \\
& \lambda_{1}=K_{m}-\frac{2}{3} \mu, \\
& d_{1}=1-\Delta_{N}, \\
& d_{2}=1-\frac{\lambda^{2} \Delta_{N}}{L^{2}}, \\
& K=\lambda+\frac{2 \mu}{3},
\end{aligned}
$$

and $K$ is the bulk modulus of background material; $K_{f}$ is the fluid bulk modulus, which can be calculated by the bulk moduli of water $\left(K_{w}\right)$, gas $\left(K_{g}\right)$, and oil $\left(K_{o}\right)$ with the corresponding saturations $S_{w}, S_{g}$, and $S_{o}$ :

$$
\frac{1}{K_{f}}=\frac{S_{w}}{K_{w}}+\frac{S_{o}}{K_{o}}+\frac{S_{g}}{K_{g}} .
$$

In (4), $L, D, \eta, \lambda_{1}, d_{1}, d_{2}, b_{0}$, and $b^{\prime}$ are the intermediate variables; $\lambda$ and $\mu$ are the Lamé parameter and the shear modulus of the background isotropic material, respectively; $\varphi$ is the total porosity of the fractured rock and $\varphi_{m}$ and $\varphi_{c}$ are the porosities of background material and fractures, respectively; $a$ is the aspect ratio of the fracture; $e$ is the fracture density; $K_{m}$ is the solid grain bulk modulus. $\Delta_{N}$ and $\Delta_{T}$ are the normal and tangential fracture weaknesses, which can be calculated by the dimensionless normal $\left(E_{N}\right)$ and tangential $\left(E_{T}\right)$ compliances of the fracture system, respectively: 


$$
\begin{aligned}
\Delta_{T} & =\frac{E_{T}}{1+E_{T}}, \\
\Delta_{N} & =\frac{E_{N}}{1+E_{N}}, \\
E_{N} & =\frac{4 e}{3 g\left[1-g+\left(k^{\prime}+4 \mu^{\prime} / 3\right) /(\pi a \mu)\right]}, \\
E_{T} & =\frac{16 e}{3\left(3-2 g+4 \mu^{\prime} / \pi a \mu\right)}, \\
g & =\frac{\mu}{\lambda+2 \mu},
\end{aligned}
$$

where $k^{\prime}$ and $\mu^{\prime}$ are the bulk and shear moduli of the fillings in the fractures, respectively.

Equations (3)-(6) provide a complete description of the stiffness coefficients of the saturated fractured HTI model. Then, the elastic and anisotropy parameters can be expressed in terms of the properties of dry (isotropy) background, fillings in fractures, and saturating fluid by substituting saturated rock stiffness coefficient equations (3)-(6) into (2):

$$
\begin{aligned}
& \alpha=\sqrt{\frac{9 L D_{4}-4 B \Delta_{N}}{9 D L \rho}}, \\
& \beta=\sqrt{\frac{\mu}{\rho}}, \\
& \varepsilon^{(V)}=\frac{12 B \Delta_{N}+9\left(D_{2}-D_{1}\right) L^{2}}{8 B-18 L D_{4}}, \\
& \delta^{(V)} \\
& =\frac{3\left[-3\left(D_{4}+D_{3}\right) L+4 B \Delta_{N}\right]\left[4 B \Delta_{N}+9 L\left(D_{4}-D_{3}-2 D T\right)\right]}{2\left(-9 D_{4} L+4 B \Delta_{N}\right)\left[4 B \Delta_{N}-9 L\left(D_{4}-D T\right)\right]}, \\
& \gamma=\frac{\Delta_{T}}{2\left(1-\Delta_{T}\right)},
\end{aligned}
$$

where

$$
\begin{aligned}
D_{1}= & \left(1-\Delta_{N}\right)\left(1-\frac{K_{f}}{K_{m}}\right), \\
D_{2}= & \left(1-\frac{\lambda^{2}}{L^{2}} \Delta_{N}\right)\left(1-\frac{K_{f}}{K_{m}}\right), \\
D_{3}= & \frac{K_{f}}{\varphi K_{m}}\left(K_{m}-\frac{2}{3} \mu\right)\left(b_{0}+\frac{K^{2} \Delta_{N}}{K_{m} L}\right) \\
& +\lambda\left(1-\Delta_{N}\right)\left(1-\frac{K_{f}}{K_{m}}\right), \\
& \frac{K_{f}}{\varphi K_{m}}\left(K_{m}+\frac{4}{3} \mu\right)\left(b_{0}+\frac{K^{2} \Delta_{N}}{K_{m} L}\right) \\
& +\left(1-\frac{\lambda^{2}}{L^{2}} \Delta_{N}\right)\left(1-\frac{K_{f}}{K_{m}}\right)(\lambda+2 \mu),
\end{aligned}
$$

$$
\begin{aligned}
& B=b_{0} u^{2} \frac{K_{f}}{\varphi K_{m}}, \\
& T=\mu\left(1-\Delta_{T}\right), \\
& \rho=(1-\varphi) \rho_{m}+\varphi\left(S_{w} \rho_{w}+S_{o} \rho_{o}+S_{g} \rho_{g}\right),
\end{aligned}
$$

and $B, T, D_{1}, D_{2}, D_{3}$, and $D_{4}$ are the intermediate variables. Then, we can obtain the elastic and anisotropy parameters for establishing the saturated HTI model if the background medium parameters of $\lambda, \mu, K_{m}, \varphi_{m}$, and $\rho_{m}$ (the background medium density), fracture system parameters of $a, e, k^{\prime}$, and $\mu^{\prime}$, and saturating fluid parameters of $K_{w}, K_{g}, K_{o}, S_{w}, S_{g}, S_{o}$, $\rho_{w}, \rho_{g}$, and $\rho_{o}$ are known.

2.2. Reflection Coefficients. To study the AVA response characteristics of the saturated HTI model, the reflection coefficients of PP- and split PS-waves can be calculated by Rüger's equations [14]:

$$
\begin{aligned}
& R_{\mathrm{PP}}=\frac{1}{2} \frac{\Delta Z}{\bar{Z}}+\frac{1}{2}\left\{\frac{\Delta \alpha}{\bar{\alpha}}-\left(\frac{2 \bar{\beta}}{\bar{\alpha}}\right)^{2} \frac{\Delta G}{\bar{G}}\right. \\
& \left.+\left[\left(\delta_{2}^{(V)}-\delta_{1}^{(V)}\right)+2\left(\frac{2 \bar{\beta}}{\bar{\alpha}}\right)^{2}\left(\gamma_{2}-\gamma_{1}\right)\right] \cos ^{2} \theta\right\} \\
& \cdot \sin ^{2} i_{1}+\frac{1}{2}\left\{\frac{\Delta \alpha}{\bar{\alpha}}+\left(\varepsilon_{2}{ }^{(V)}-\varepsilon_{1}{ }^{(V)}\right) \cos ^{4} \theta\right. \\
& \left.+\left(\delta_{2}^{(V)}-\delta_{1}^{(V)}\right) \sin ^{2} \theta \cos ^{2} \theta\right\} \sin ^{2} i_{1} \tan ^{2} i_{1}, \\
& R_{\mathrm{PS} 1}=-\frac{1}{2} \frac{\Delta \rho}{\bar{\rho}} \frac{\sin i_{1}}{\cos j_{1}}-\frac{\bar{\beta}}{\bar{\alpha}}\left(\frac{\Delta \rho}{\bar{\rho}}+2 \frac{\Delta \beta}{\bar{\beta}}\right) \sin i_{1} \cos i_{1} \\
& +\left(\frac{\bar{\beta}}{\bar{\alpha}}\right)^{2}\left(2 \frac{\Delta \beta}{\bar{\beta}}+\frac{\Delta \rho}{\bar{\rho}}\right) \frac{\sin ^{3} i_{1}}{\cos j_{1}}, \\
& R_{\mathrm{PS} 2}=-\frac{1}{2}\left(\frac{\Delta \rho}{\rho}\right) \frac{\sin i_{1}}{\cos j_{1}}-\frac{\bar{\beta}}{\bar{\alpha}}\left\{\frac{\Delta \rho}{\rho}\right. \\
& \left.+2\left(\frac{\Delta \beta}{\bar{\beta}}+\gamma_{1}-\gamma_{2}\right)\right\} \sin i_{1} \cos i_{1}+\left(\frac{\bar{\beta}}{\bar{\alpha}}\right)^{2} \\
& \cdot\left\{2\left(\frac{\Delta \beta}{\bar{\beta}}+\gamma_{1}-\gamma_{2}\right)+\frac{\Delta \rho}{\rho}\right\} \frac{\sin ^{3} i_{1}}{\cos j_{1}} \\
& +\left[\left(\frac{\bar{\alpha}^{2}}{2\left(\bar{\alpha}^{2}-\bar{\beta}^{2}\right) \cos j_{1}}-\frac{\bar{\alpha} \bar{\beta} \cos i_{1}}{2\left(\bar{\alpha}^{2}-\bar{\beta}^{2}\right)}\right)\right. \\
& \left.\cdot\left(\delta_{2}^{(V)}-\delta_{1}^{(V)}\right)\right] \sin i_{1}
\end{aligned}
$$




$$
\begin{aligned}
& +\left[\frac{\bar{\alpha} \bar{\beta} \cos i_{1}}{\left(\bar{\alpha}^{2}-\bar{\beta}^{2}\right)}\left(\delta_{2}^{(V)}-\delta_{1}{ }^{(V)}+\varepsilon_{1}{ }^{(V)}-\varepsilon_{2}{ }^{(V)}\right)\right] \\
& \cdot \sin ^{3} i_{1}-\left[\left(\frac{\bar{\alpha}^{2}}{\left(\bar{\alpha}^{2}-\bar{\beta}^{2}\right) \cos j_{1}}\right)\right. \\
& \left.\cdot\left(\delta_{2}{ }^{(V)}-\delta_{1}^{(V)}+\varepsilon_{1}^{(V)}-\varepsilon_{2}^{(V)}\right)\right] \sin ^{3} i_{1} \\
& +\left[\left(\frac{\bar{\beta}^{2}}{2\left(\bar{\alpha}^{2}-\bar{\beta}^{2}\right) \cos j_{1}}\right)\left(\delta_{1}^{(V)}-\delta_{2}{ }^{(V)}\right)\right] \sin ^{3} i_{1} \\
& +\left[\left(\frac{\bar{\beta}^{2}}{\left(\bar{\alpha}^{2}-\bar{\beta}^{2}\right) \cos j_{1}}\right)\right. \\
& \left.\cdot\left(\delta_{2}{ }^{(V)}-\delta_{1}^{(V)}+\varepsilon_{1}^{(V)}-\varepsilon_{2}^{(V)}\right)\right] \sin ^{5} i_{1},
\end{aligned}
$$

where

$$
\begin{aligned}
\bar{\alpha} & =\frac{1}{2}\left(\alpha_{1}+\alpha_{2}\right), \\
\Delta \alpha & =\alpha_{2}-\alpha_{1}, \\
\bar{\beta} & =\frac{1}{2}\left(\beta_{1}+\beta_{2}\right), \\
\Delta \beta & =\beta_{2}-\beta_{1}, \\
\bar{\rho} & =\frac{1}{2}\left(\rho_{1}+\rho_{2}\right), \\
\Delta \rho & =\rho_{2}-\rho_{1}, \\
Z & =\rho \alpha, \\
G & =\rho \beta^{2} .
\end{aligned}
$$

Here, $i_{1}$ is the incidence phase angle of the P-wave; $j_{1}$ is the emergence phase angle of the S1-wave; $i_{1}$ and $j_{1}$ follow Snell's Law [14]; $\theta$ is the azimuth angle of the P-wave. The subscripts " 1 " and " 2 " denote parameters of the upper and lower strata, respectively. Equations (9)-(10) relate the elastic and anisotropy parameters with the PP- and split PS-wave reflection coefficients.

\section{Model Analysis}

The goal of this study was to analyze the effect of fracture density on the AVA responses of the fractured-shale model. We performed forward simulations based on the theoretical model consisting of an isotropic overburden and a fractured stratum considering the same parameters for the background overburden (Table 1). For the fractured stratum, the physical quantities of the fracture system and the saturating fluids are shown in Table 1. Following the procedure shown in Figure 2, we modeled fractured shale (Table 2) and calculated the reflection coefficients of the PP- and split PS-waves (Figure 3). Here, fluids considered in the fractured shale only include gas and water. As shown in Table 2, with fracture density increasing from 0 to 0.4 , the vertical $\mathrm{P}$ - and S-wave velocities decreased by about $4.5 \%$ and $2.8 \%$, respectively; density decreased by about $8.6 \% . \varepsilon^{(V)}$ and $\delta^{(V)}$ had negative values, and $\gamma$ was positive, which are physically reasonable [14].

3.1. AVA Analysis. As shown in Figure 3, the curves of variations in the reflection coefficients of the PP- or PS2waves are sharper than that of the PS1-wave. Figure 3(a) shows that the reflection coefficients of PP-wave increase with increasing incidence angle and then decrease significantly at incidence angles larger than $50^{\circ}$. Furthermore, the fluctuation of the PP-wave reflection coefficient curve is more obvious at high fracture density. This is because, in the lower fractured stratum, $\alpha, \beta, \rho, \varepsilon^{(V)}$, and $\delta^{(V)}$ decrease and $\gamma$ increases as fracture density increases. Moreover, differences in elastic and anisotropy parameters between the upper and lower strata increase. Figures 3(b) and 3(c) show that the variation trends of the reflection coefficients of the PS1- and PS2-waves are consistent. They increase first and then decrease with increasing incidence angle. In addition, variation rate of the reflection coefficient increases at higher fracture densities. There are two intersections between the reflection coefficient curves of the PS1- and PS2-waves with one common intersection at the incidence angle of $0^{\circ}$. For the PS1-wave, the other intersection is near the incidence angle of $65^{\circ}$, and for the PS2-wave, the other intersection is at the incidence angle of $55^{\circ}$. The reflection coefficients of the PS2-wave vary from positive to negative values, but those of the PS1-wave remain positive.

We compared AVA responses of the fractured-shale model with those of shale without fractures. The shale without fractures can be considered as a type of isotropic medium model, for which anisotropy parameters are zero. In addition, in an isotropic medium model, the shear wave will not be split. For comparison, we calculated relative differences in the reflection coefficients of the PP-wave between the fracturedshale and isotropic models (Figure 4(a)). The PS-wave reflection coefficient of the isotropic model was the same as the PS1-wave reflection coefficient of the fractured-shale model. Therefore, only the calculated relative differences between the PS-wave reflection coefficients of the isotropic model and the PS2-wave reflection coefficients of the fractured-shale model are shown in Figure 4(b). 


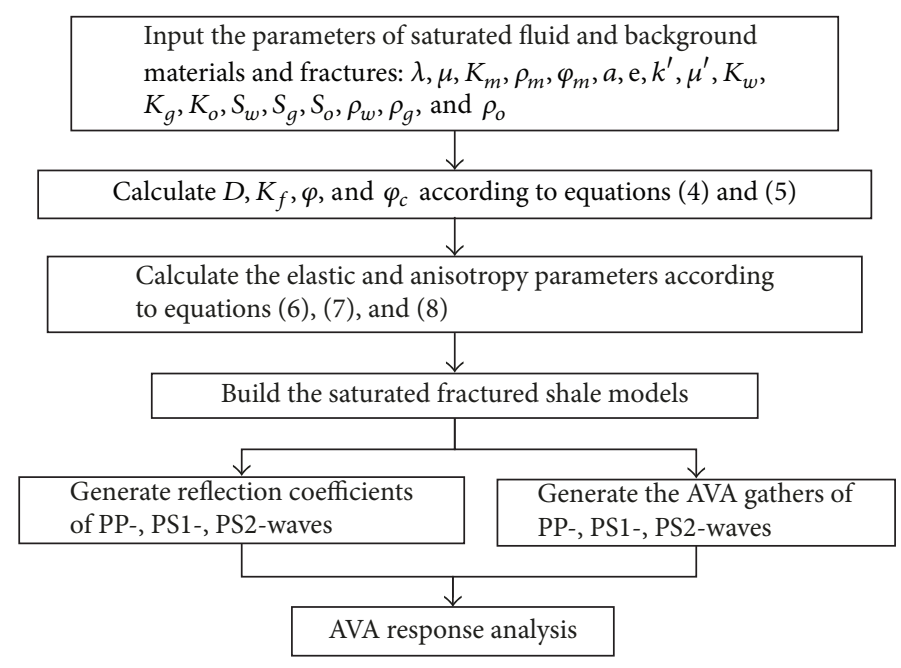

FIGURE 2: Flowchart of the fractured-shale modeling and AVA response analysis.

TABLE 1: Parameters of the background medium, fractures, and fluids.

\begin{tabular}{|c|c|c|c|c|c|}
\hline Fluid parameters & Values & Background medium parameters & Values & Fracture parameters & Values \\
\hline$K_{w}(\mathrm{GPa})$ & 2.38 & $K_{m}(\mathrm{GPa})$ & $19.08-17.68$ & $k^{\prime}(\mathrm{GPa})$ & $9.18 \mathrm{GPa}$ \\
\hline$K_{g}(\mathrm{GPa})$ & 0.02 & $\rho_{m}\left(\mathrm{~g} / \mathrm{cm}^{3}\right)$ & 2.51 & $\mu^{\prime}(\mathrm{GPa})$ & $6.03 \mathrm{GPa}$ \\
\hline$\rho_{w}\left(\mathrm{~g} / \mathrm{cm}^{3}\right)$ & 1.09 & $\mu(\mathrm{GPa})$ & $11.38-10.54$ & $e$ & $0.01-0.40$ \\
\hline$\rho_{g}\left(\mathrm{~g} / \mathrm{cm}^{3}\right)$ & 0.14 & $\lambda$ & $11.38-10.54$ & $a$ & 0.01 \\
\hline$S_{w}$ & $50 \%$ & $\varphi_{m}$ & $10 \%$ & & \\
\hline$\underline{S_{g}}$ & $50 \%$ & & & & \\
\hline
\end{tabular}

TABLE 2: Different fracture density models of shale.

\begin{tabular}{lccccrr}
\hline Fracture density & $\alpha(\mathrm{km} / \mathrm{s})$ & $\beta(\mathrm{km} / \mathrm{s})$ & $\rho\left(\mathrm{g} / \mathrm{cm}^{3}\right)$ & $\varepsilon^{(V)}$ & $\delta^{(V)}$ & 0 \\
\hline 0 & 3.85 & 2.21 & 2.5 & 0 & 0 & 0.003 \\
0.01 & 3.839 & 2.217 & 2.311 & -0.003 & -0.005 \\
0.10 & 3.798 & 2.201 & 2.304 & -0.030 & -0.050 & 0.029 \\
0.20 & 3.756 & 2.183 & 2.297 & -0.055 & -0.090 \\
0.40 & 3.679 & 2.149 & 2.283 & -0.097 & -0.152 & 0.057 \\
\hline
\end{tabular}

As shown in Figure 4(a), with increasing incidence angle, the absolute value of the relative differences initially increase, then decrease, and dramatically increase towards the end. Although the velocities and densities of the two models are the same, changes in anisotropic parameters will greatly affect the reflection coefficient. In Figure 4(b), the relative difference between the two models becomes more apparent at higher fracture density.

In the fractured-shale model, within incidence angles of $40^{\circ}$, higher fracture density can lead to large changes in the PS2-wave reflection coefficients but small changes in the $\mathrm{PP}$-wave reflection coefficients. However, at incidence angles over $55^{\circ}$, the reflection coefficients of both the PP- and PSwaves change dramatically.

3.2. AVA Gathers Analysis. According to the model parameters in Table 2, AVA gathers were synthesized by convolution using the Ricker wavelet with a dominant frequency of $40 \mathrm{~Hz}$ and the reflection coefficients shown in Figure 3. AVA gathers of the PP-wave with different fracture densities are shown in Figures 5(a), 5(b), 5(c), and 5(d). The reflection amplitudes are enhanced with increasing fracture density, especially at large incidence angles over $60^{\circ}$. For small fracture density, as shown in Figure 5(a), the reflection amplitudes decrease with 

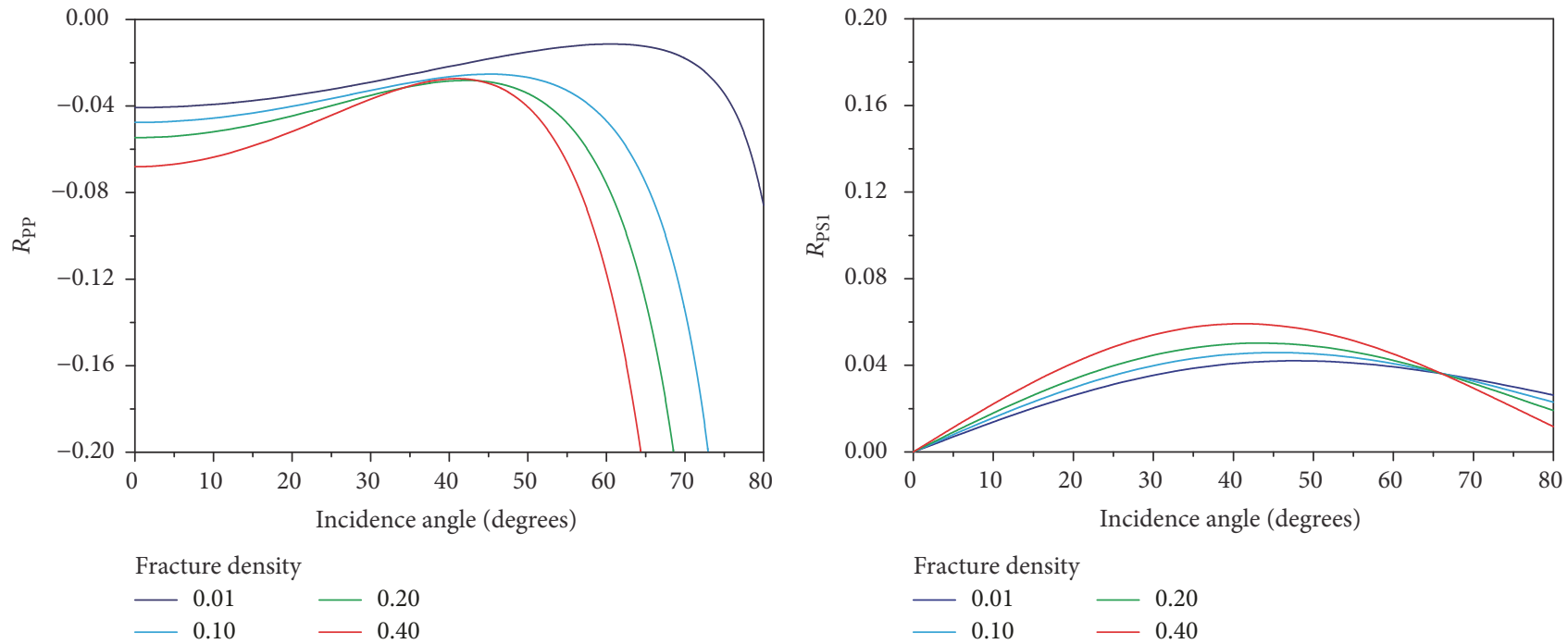

$0.01 \quad 0.20$
$-0.10 \quad 0.40$

Fracture density

(a)

0.20

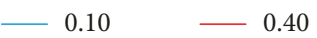

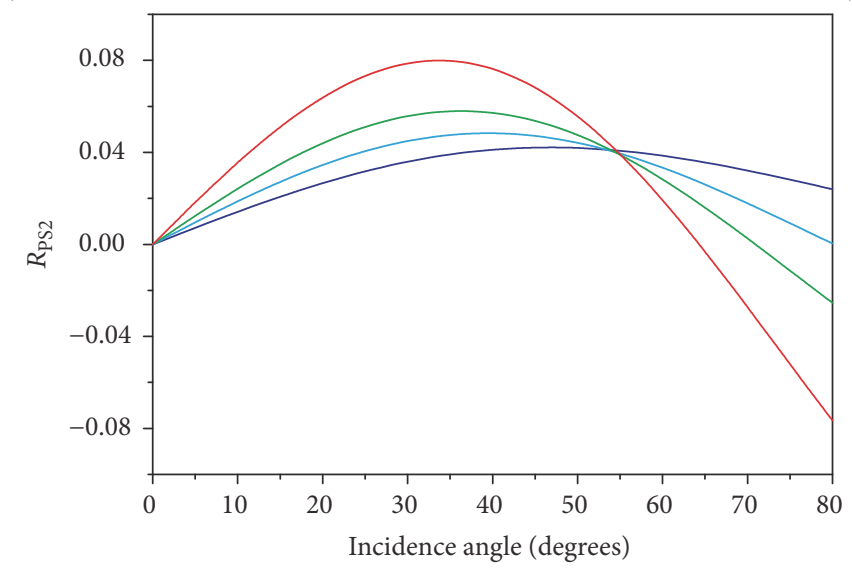

(b)

Fracture density

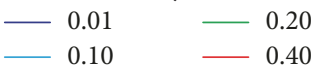

(c)

FIgURE 3: AVA response curves of the PP-wave (a), PS1-wave (b), and PS2-wave (c) as a function of incidence angles and fracture densities.
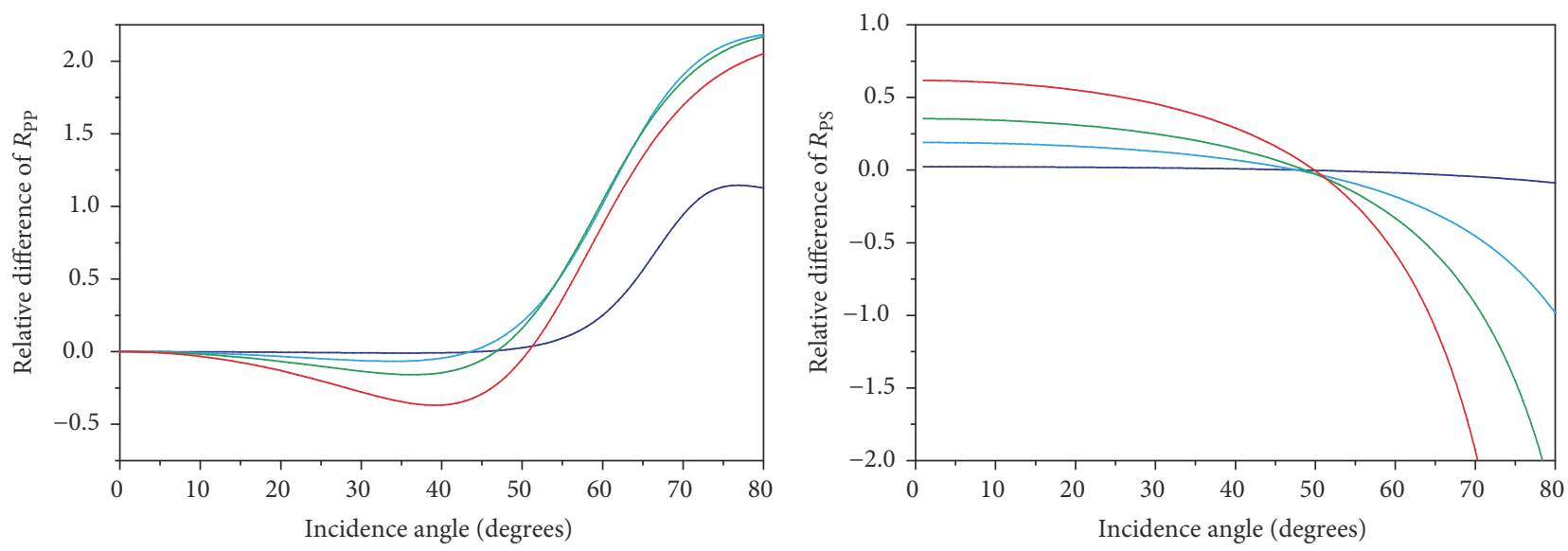

Fracture density

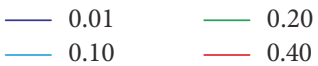

Fracture density

$-0.01-0.20$

(a)

(b)

FIGURE 4: Relative differences in the reflection coefficients of the PP-wave (a) and PS-wave (b) between the fractured-shale model and the isotropic model. 


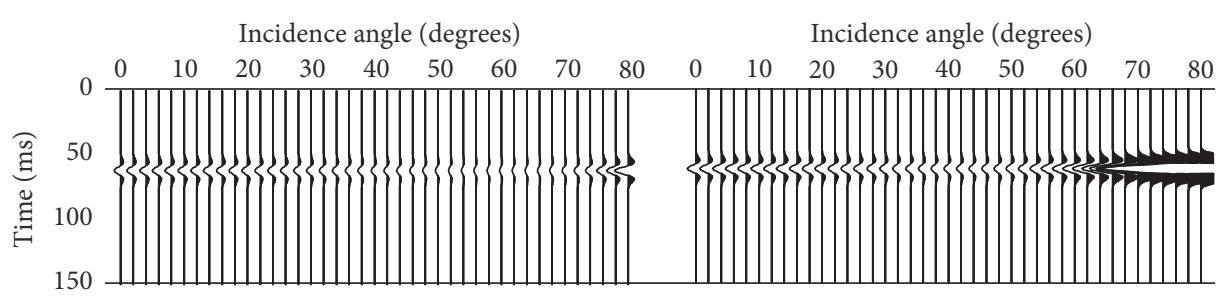

(a) PP-wave, $\mathrm{e}=0.01$

(b) PP-wave, e = 0.1

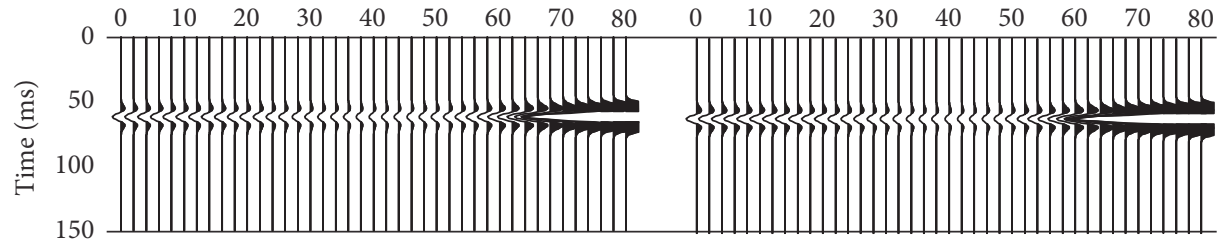

(c) PP-wave, e = 0.2

(d) PP-wave, e = 0.4

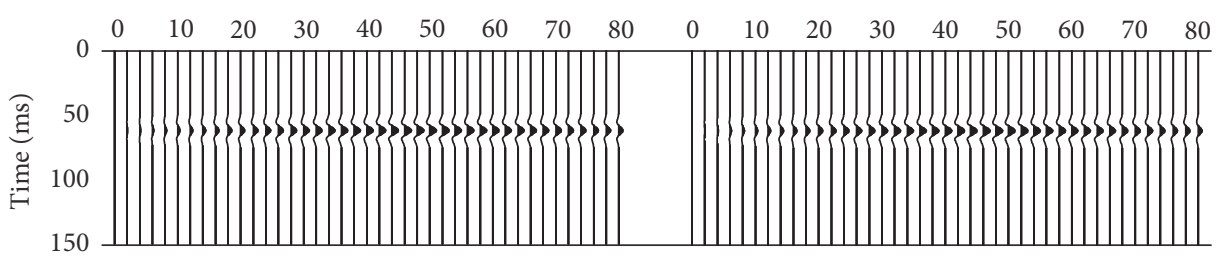

(e) PS1-wave, $\mathrm{e}=0.01$

(f) PS1-wave, e = 0.1

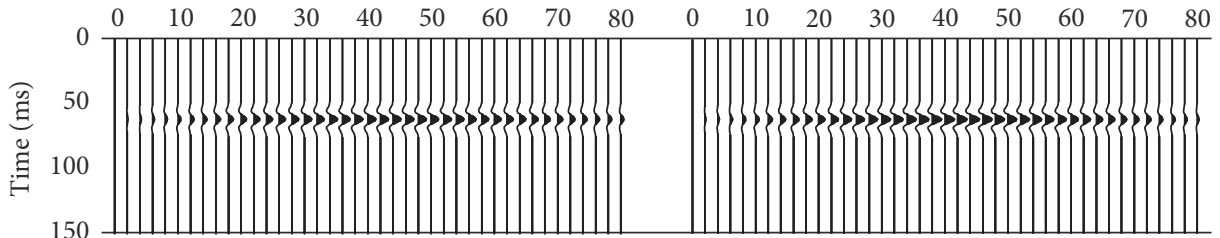

(g) PS1-wave, e = 0.2

(h) PS1-wave, e = 0.4

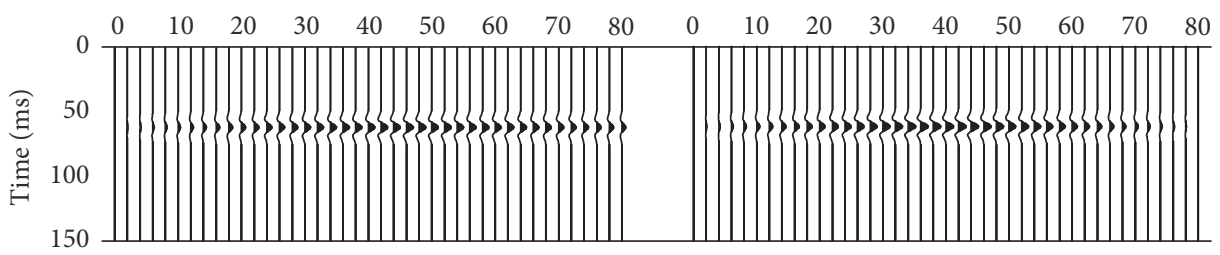

(i) PS2-wave, $\mathrm{e}=0.01$

(j) PS2-wave, e = 0.1

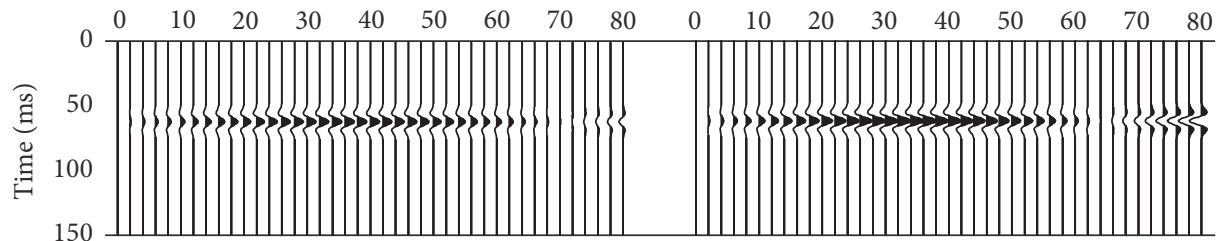

(k) PS2-wave, e $=0.2$

(l) PS2-wave, e = 0.4

FIGURE 5: AVA gathers of PP-, PS1-, and PS2-waves as a function of different fracture densities.

increasing incidence angle. As shown in Figures 5(b), 5(c), and 5(d), the PP-wave reflection amplitudes first increase and then decrease with increasing incidence angle. The PS1-wave AVA gathers in Figures 5(e), 5(f), 5(g), and 5(h) clearly show strong reflection amplitudes at intermediate incidence angles. However, there was no significant change in AVA gathers with varying fracture densities. In Figures 5(i), 5(j), 5(k), and 5(l), the PS2-wave AVA gathers show strong reflection amplitudes at intermediate incidence angles. However, polarity reversal occurs at fracture densities of 0.2 and 0.4 .

\section{Conclusions}

In this study, we determined the relationship between Gurevich's fluid theory and Thomsen's anisotropy parameters for the saturated-fractured-shale model and simulated PP- and 
split PS-wave AVA responses. From analyses of the reflection coefficients of the saturated-fractured-shale and isotropic models, we found that anisotropy parameters increase with increasing fracture density, and the difference in reflection coefficients become more prominent. The reflection coefficients of the two models were distinct at incidence angles higher than $55^{\circ}$. The differences in reflection coefficients between the two models grew wider as fracture density increased. Moreover, the differences in reflection coefficients were more dramatic for PP- or PS2-waves than for PS1waves. According to the synthesized AVA gathers, we found that polarity reversal occurs only in PS2-wave AVA gathers with high fracture density. The modeling of water-filled or oil-filled fractures remains a major unresolved issue. The velocities and densities of water and oil are close, and thus their corresponding influences on elastic and anisotropy parameters are similar. Therefore, discriminating the AVA response anomaly caused by water and oil is still a problem. Nevertheless, the proposed simulations of the fractured-shale model will lay the foundation for further studies on the wavefield characteristics of actual fractured reservoirs.

\section{Conflicts of Interest}

The authors declare that they have no conflicts of interest.

\section{Acknowledgments}

The authors greatly appreciate the support of the Natural Science Foundation of China (41574126 and 41425017) and the Fundamental Research Funds for the Central Universities (2-9-2017-452). They would like to thank Editage (https://www.editage.com) for English language editing.

\section{References}

[1] J. P. Castagna, H. W. Swan, and D. J. Foster, "Framework for AVO gradient and intercept interpretation," Geophysics, vol. 63, no. 3, pp. 948-956, 1998.

[2] P. Avseth, A. Draege, A.-J. van Wijingaarden, T. A. Johansen, and A. Jørstad, "Shale rock physics and implications for AVO analysis: a North Sea demonstration," The Leading Edge, vol. 27, no. 6, pp. 788-797, 2008.

[3] L. Thomsen, "Elastic anisotropy due to aligned cracks in porous rock," Geophysical Prospecting, vol. 43, no. 6, pp. 805-829, 1995.

[4] S. Crampin, "Seismic-wave propagation through a cracked solid: polarization as a possible dilatancy diagnostic," The Geophysical Journal of the Royal Astronomical Society, vol. 53, no. 3, pp. 467-496, 1978.

[5] S. Crampin, "Evaluation of anisotropy by shear-wave splitting.," Geophysics, vol. 50, no. 1, pp. 142-152, 1985.

[6] A. Rüger and I. Tsvankin, "Azimuthal variation of AVO response for fractured reservoirs: 65th annual international meeting," SEG Expanded Abstracts, pp. 1103-1106, 1995.

[7] A. Rüger and I. Tsvankin, "Using AVO for fracture detection: analytic basis and practical solutions," The Leading Edge, vol. 16, no. 10, p. 1429, 1997.

[8] A. Rüger, "P-wave reflection coefficients for transversely isotropic models with vertical and horizontal axis of symmetry," Geophysics, vol. 62, no. 3, pp. 713-722, 1997.
[9] A. Rüger, "Variation of P-wave reflectivity with offset and azimuth in anisotropic media," Geophysics, vol. 63, no. 3, pp. 935-947, 1998.

[10] M. A. Pérez, R. L. Gibson, and M. N. Toksöz, "Detection of fracture orientation using azimuthal variation of P-wave AVO responses," Geophysics, vol. 64, no. 4, pp. 1253-1265, 1999.

[11] D. Gray and K. Head, "Fracture detection in Manderson field: a 3-D AVAZ case history," The Leading Edge, vol. 19, no. 11, pp. 1214-1221, 2000.

[12] S. A. Hall and J.-M. Kendall, "Fracture characterization at Valhall: application of P-wave amplitude variation with offset and azimuth (AVOA) analysis to a 3D ocean-bottom data set," Geophysics, vol. 68, no. 4, pp. 1150-1160, 2017.

[13] A. Rüger, "Analytic insight into shear-wave AVO for fractured reservoirs: 66th annual international meeting," Society of Exploration Geophysicists, pp. 159-185, 1996.

[14] A. Rüger, "Reflection coefficients and azimuthal AVO analysis in anisotropic media," Society of Exploration Geophysicists, pp. 63-181, 2002.

[15] J. A. Hudson, "A higher order approximation to the wave propagation constants for fractured solid," Geophysical Journal International, vol. 87, no. 1, pp. 265-274, 1986.

[16] M. Schoenberg, "Reflection of elastic waves from periodically stratified media with interfacial slip," Geophysical Prospecting, vol. 31, no. 2, pp. 265-292, 1983.

[17] L. Thomsen, "Weak elastic anisotropy," Geophysics, vol. 51, no. 10, pp. 1954-1966, 1986.

[18] L. Thomsen, "Converted-wave reflection seismology over inhomogeneous, anisotropic media," Geophysics, vol. 64, no. 3, pp. 678-690, 1999.

[19] M. Chapman, "Modeling the effect of multiple sets of mesoscale fractures in porous rock on frequency-dependent anisotropy," Geophysics, vol. 74, no. 6, pp. 97-103, 2009.

[20] R. Cardona, "Two theories for fluid substitution in porous rocks with aligned fractures: 72nd annual international meeting," SEG Expanded Abstracts, pp. 173-176, 2002.

[21] B. Gurevich, "Elastic properties of saturated porous rocks with aligned fractures," Journal of Applied Geophysics, vol. 54, no. 3-4, pp. 203-218, 2003.

[22] S. Crampin, "Suggestions for a consistent terminology for seismic anisotropy," Geophysical Prospecting, vol. 37, no. 7, pp. 753-770, 1989 .

[23] L. Brown and B. Gurevich, "Frequency-dependent seismic anisotropy of porous rocks with penny-shaped cracks," Exploration Geophysics, vol. 35, no. 2, pp. 111-115, 2004. 




Advances in

Operations Research

\section{-n-m}
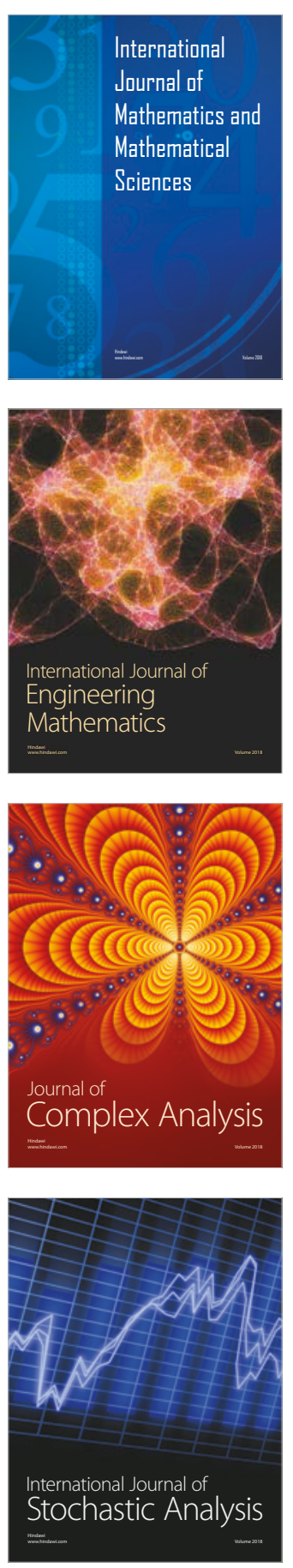
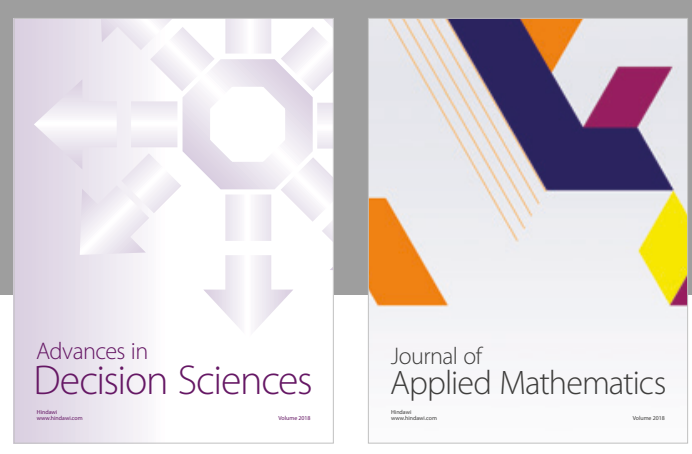

Journal of

Applied Mathematics
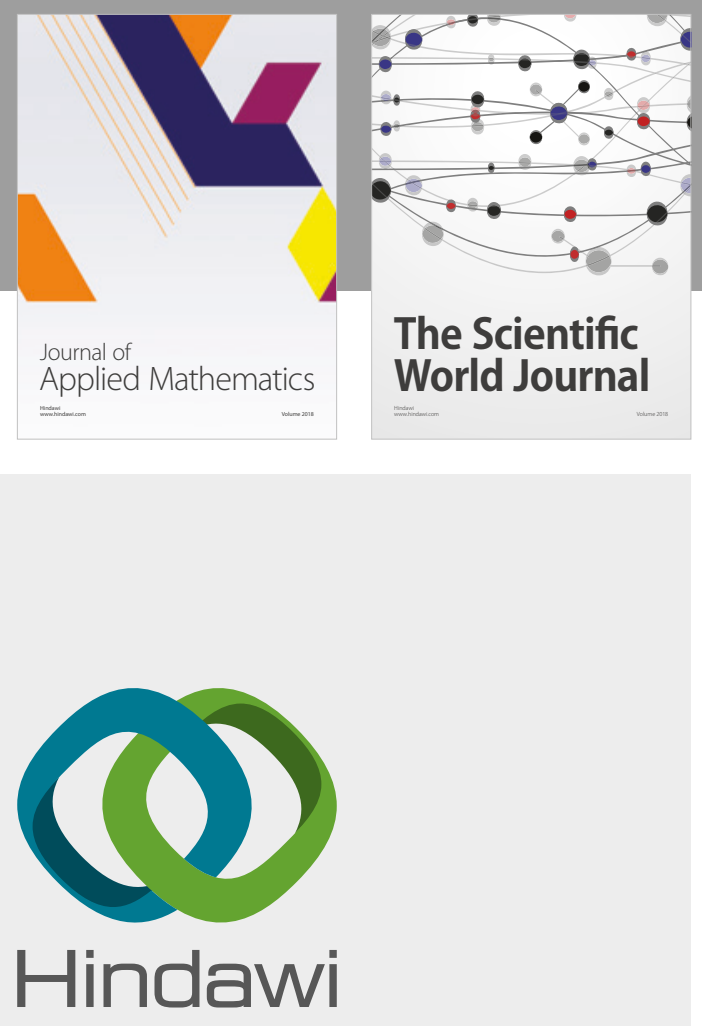

Submit your manuscripts at

www.hindawi.com

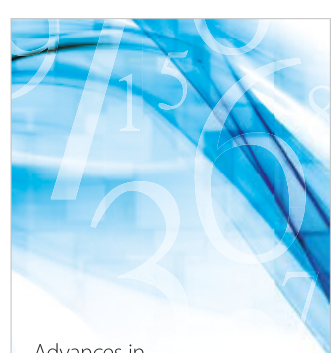

Advances in
Numerical Analysis
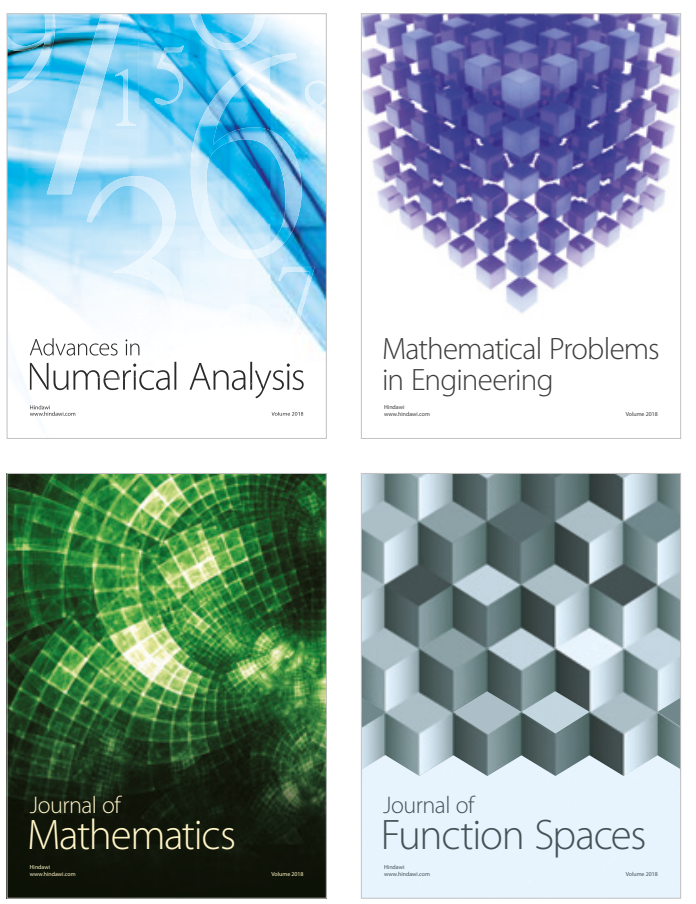

Mathematical Problems in Engineering



International Journal of

Differential Equations

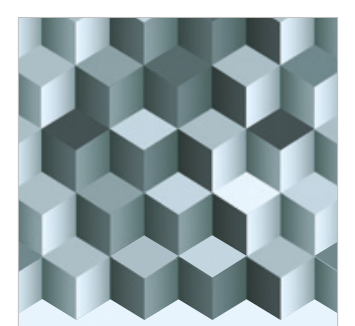

Journal of

Function Spaces

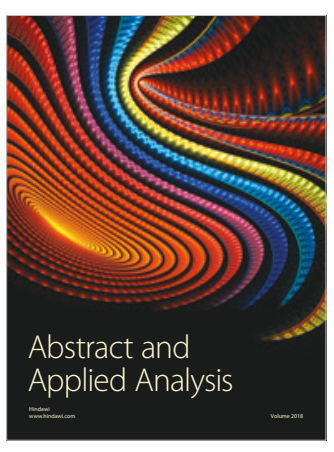

The Scientific

World Journal

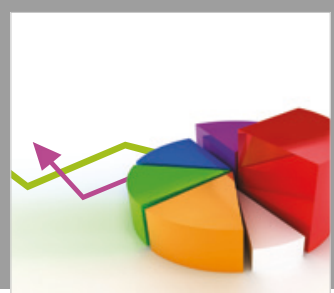

Journal of

Probability and Statistics
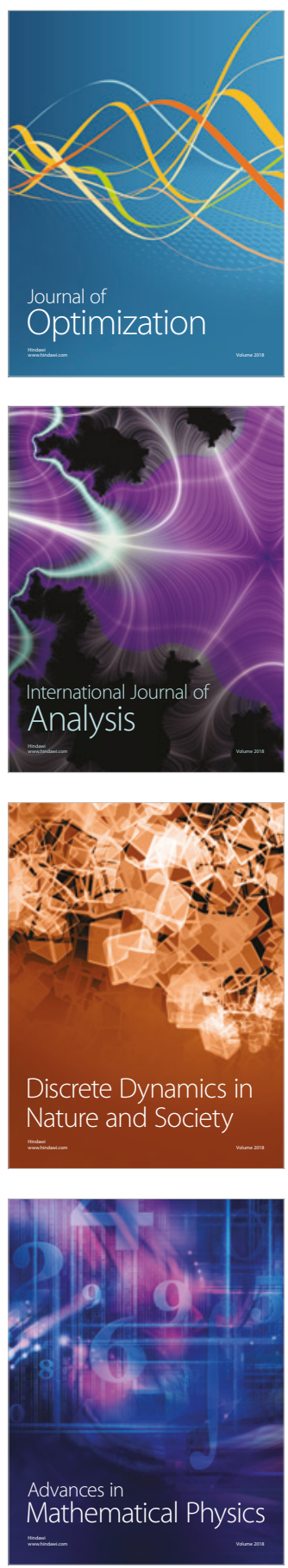